\title{
Study on Flexural Properties of Tubular Underwater Inflated Structures Filled with Hydrogel Beads
}

\author{
Yanjun $\mathrm{Li}^{1}$, Radivoje Stankovic ${ }^{2}$, Bing Ouyang${ }^{1}$, Tsung-chow $\mathrm{Su}^{2}$ \\ 1. Harbor Branch Oceanographic Institute, Florida Atlantic University, Fort Pierce, FL, 34946, USA \\ 2. Department of Ocean and Mechanical Engineering, Florida Atlantic University, Boca Raton, FL, 33431, USA \\ E-mail: yli2013@fau.edu; rstankovic2015@fau.edu; bouyang@fau.edu; su@fau.edu
}

Received: 5 September 2018; Accepted: 26 September 2018; Available online: 20 February 2019

\begin{abstract}
Underwater inflatable structures (UISs) are distributed in an initial folded state for ease of transportation and deployment. They are able to morph into their intended geometry upon arrival at their destination. This morphing/inflation process can be driven by hydrogel beads, which swell when they contact water. In this research, we study the physical characteristics of tubular UISs with different configurations (length, slenderness, beads density). We fabricated sets of tubular UISs with nylon sleeves filled with different amounts of hydrogel beads and built a flexural test platform mounted on the test tank for the bending experiment. This work provides the experimental Young's modulus of the tubular UISs under elastic and plastic deformations, and also discusses how the different configurations contribute to the tubular UIS's stiffness.
\end{abstract}

Keywords: Underwater inflatable structure; Inflated structure; Flexural property; Hydrogel beads.

\section{Introduction}

As much of the Earth's ocean remains unexplored, there is a need for new structural materials suitable for these environments, and that can operate under significant surrounding pressure environment. The concept of underwater inflatable structures (UISs) was initially proposed [1] to build morphing oceanic structures as an underwater architecture to aid with ocean exploration and research work. UISs initially come in a folded state for easy transportation and manipulation. The structures are able to morph into their intended geometry and maintain their inflated status via hydraulic approach. The hydraulic approach employs an underwater pump to inject the surrounding water into the UIS which effectively maintains the inflated status, giving the structure an inner and outer pressure difference. There is a practical alternate approach to achieve the same effect using hydrophilic infill materials, like hydrogel products.

Hydrogel products are polymer materials which can absorb water and retain a significant fraction of water compared to the polymer volume. [2] There are many applications for hydrogel product utilization including agriculture [3], sealing [4], food additives [5], artificial tissue [6, 7], and pharmaceuticals [8]. In our study, we utilize the physical characteristic of hydrogel beads to maintain the UIS's desired geometry.

In this paper, we focus on the inflated structure's mechanical properties with different configurations. The fabrication of the tubular UIS and analysis methods are outlined in section 2. The experimental setup and results are presented respectively in sections 3 and 4 . Section 5 provides the results discussion and concluding remarks.

\section{Materials and methods}

The tubular UIS contains permeable fabrics and hydrogel beads. The permeable fabrics form a tubular, enclosed space to store the unactivated hydrogel beads. Upon coming in contact with water, the hydrogel beads expand, which drives the tubular UIS to morph into its final shape. The morphed tubes are taken as prepared samples for bending tests to obtain flexural properties.

This section presents the hydrogel bead properties, materials selection, the tubular UIS dimensional calculation, and the formulations of the modulus of elasticity.

\subsection{Materials properties and selection}

Hydrogel beads expand when they come in contact with water; this physical property contributes to UIS morphing. The swollen hydrogel beads can maintain their increased size allowing the morphed structure to maintain its geometry. The swollen beads can shrink back down to their initial size after dehydration, which makes the UIS recoverable. The 
hydrogel beads in different states are shown in Figure1: green is dry/unactivated, yellow is swollen, and the blue and red show two stages of dehydration). The beads are placed on a $1 \mathrm{~cm}$ by $1 \mathrm{~cm}$ grid paper. The green bead shows the initial state with a diameter of $2.15 \mathrm{~mm}$; the yellow bead is swollen with water and features a diameter of $14.71 \mathrm{~mm}$; the blue bead shows the bead after three days in the dehydration process, with a diameter of $6.86 \mathrm{~mm}$; the red bead shows the status after two weeks of dehydration, with a diameter of $3.40 \mathrm{~mm}$. The hydrogel beads swollen-dry expansion ratio is 320 times in volume.

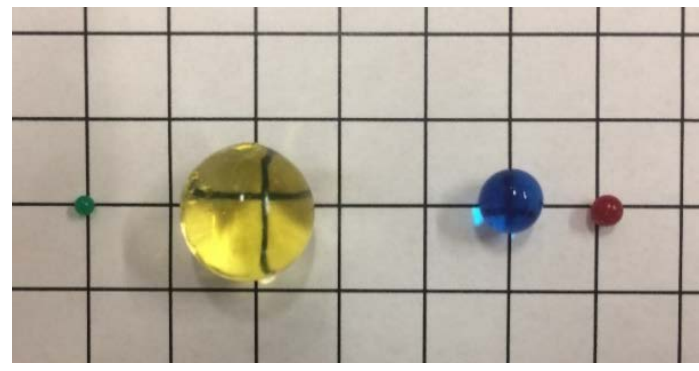

Figure 1. Hydrogel beads in dry, swollen, and two dehydration states, respectively

The beads are capable of undergoing a dehydration process wherein they shrink back down to a size only slightly larger than their initial state. This property contributes to building a recoverable UIS structure suitable for prototype study and repetitive operation. The synthetic hydrogels' responses to multiple stimuli, such as temperature, electric/magnetic field, light, pressure, $\mathrm{PH}$, etc, are known. However, we plan to study flexural properties of the tubular UISs filled with different amounts of beads. To that end, we need to consider the physical properties of any single type of easily accessible hydrogel beads, such as "Magic beads" sold readily on the market. Another benefit of hydrogel beads is that their morphing process does not consume energy, and moreover, the swollen beads are not toxic. These facts contribute to building an eco-friendly structure.

The tubular UISs require the swollen beads to fill their internal space to support and maintain the desired geometry. It is obvious that the UISs require breathable fabric allow the hydrogel bead to be exposed to surrounding water to maintain their swollen state. However, the hydrogel beads become fragile during the swelling process. The interaction between the beads themselves and the enclosing fabrics can crush the swollen beads or break the tube. The crushed beads particles leak out of the tube through the thread spacing and broken seam. Therefore, we need to select a breathable fabric that's easy to sew and features good tensile strength. Based on our previous studies [9], the nylon abrasion/hose sleeve was selected to fabricate the tubular UISs. The sleeve is woven from thousands of nylon filaments into a self-renewing, abrasion-resistant fabric sleeve [10]. This nylon sleeve is capable of withstanding bead expansion and is convenient for tubular structure fabrication.

\subsection{Calculations and Methods}

The flexural property study requires a beam with a simply supported constraint. Figure 2 illustrates the simple supported beam under a concentrated force at the mid-span, where $D$ is the deflection, beam span is $L$, the applied force is $P$, the outside beam circular cross section diameter is $d_{1}$, the inner side of the Nylon fabric circular cross section diameter is $d_{2}$, and the thickness of the Nylon fabric is $t$.
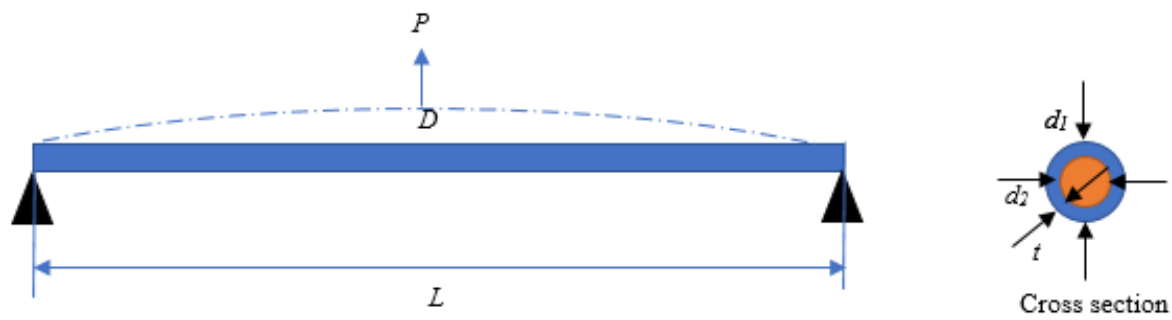

Figure 2. Beam bending diagram

As the tubular UIS is filled with homogeneous hydrogel beads [11], we can consider the tubular UIS as a continuous and homogeneous beam. Although the beam is typical composite beam and the nylon material is anisotropic, we can still apply the beam bending theory for the tubular UIS for the initial study; the maximum deflection is calculated in Equation (1):

$$
D=\frac{P L^{3}}{48 \overline{E I}}
$$


where $\overline{E I}=E_{N} I_{N}+E_{H} I_{H}=E_{E} I . E_{N}$ is Elastic modulus of nylon fabric, $I_{N}$ is Moment of Inertia of nylon fabric cross section, $E_{H}$ is Elastic modulus of hydrogel beads, $I_{H}$ is Moment of Inertia of hydrogel beads beam cross-section, the $E_{E}$ is the equivalent elastic modulus of one material beam and $I$ is the Moment of Inertia of tubular UIS cross-section.

The beam stress [12] on the $\mathrm{x}$-axis is:

$$
\sigma_{x}(y)=-\frac{M_{b}(x) y}{I}
$$

where $M_{b}(x)$ is the moment acting on the beam at $x ; y$ indicates the location of the beam element in the $y$-direction.

The maximum stress occurs at the top surface of the beam, yielding:

$$
\sigma_{\max }=-\frac{-P \cdot \frac{L}{2} \cdot \frac{d_{1}}{2}}{I}=\frac{\frac{P L d_{1}}{4}}{\frac{\pi d_{1}^{4}}{64}}=\frac{16 P L}{\pi d_{1}^{3}}
$$

The strain on the outer surface is:

$$
\varepsilon_{f}=\frac{3 D d_{1}^{2}}{L^{3}}
$$

This beam is considered a composite beam with a tubular nylon pipe filled with hydrogel beads. The volume of the swollen hydrogel beads is supposed to be greater than the volume of the nylon sleeve enclosure. This volume difference results in the surface tension of fabric to maintain beam stiffness. A greater volume difference will lead to a stiffer tubular UIS. As a result, the elastic modulus of hydrogel beads varies with different configurations. Putting Equation (1) and the definition of $E_{E}$ together yields:

$$
E_{E} I=\frac{P L^{3}}{48 D}=E_{N} I_{N}+E_{H} I_{H}
$$

Substituting the Moment of Inertia of $I, I_{N}$ and $I_{H}$, we get:

$$
E_{H}=\frac{4 P L^{3}}{3 D \pi d_{2}^{4}}-\frac{8 E_{N} d_{1}^{3} t}{d_{2}^{4}}
$$

\section{Experiments}

We prepared the tubular beam specimens as regular beams with a length-to-diameter ratio of 15:1 referring to ASTM D790-03 [13]. Beams with three different diameters (tube1-18mm, tube2-31.8mm and tube3-40.4mm) are prepared using different size tubes; the corresponding lengths for the three beams are $270.5 \mathrm{~mm}$, $476.3 \mathrm{~mm}$, and $605.8 \mathrm{~mm}$. We prepared five specimens for each tube set to fill with different amounts of hydrogel beads to obtain various beam stiffnesses. The enclosing volumes for each set of tubes are: tube1-69.10 $\mathrm{cm}^{3}$; tube2- $377.01 \mathrm{~cm}^{3}$ and tube3-776.02 $\mathrm{cm}^{3}$. One hydrogel bead free expansion volume is about $1.66 \mathrm{~cm}^{3}$ based on measurement of the swollen bead dimension in section 2 . We assume each hydrogel bead occupies $1 \mathrm{~cm}^{3}$ with the consideration of the softness and frangibility of the swollen beads. As discussed in section 2, the volume difference of the swollen beads and tube enclosure volume contribute to building a beam with stiffness. For each tube set, we started with twice as many beads as the tube enclosure volume for the first tubes, then added beads equivalent to the tube enclosure volume for the following tube specimens. In this way, we can showcase five different bead densities within one set. The number of hydrogel beads for each of the tube specimen is shown in Table 1. We placed different numbers of dry hydrogel beads in the sealed tube, shown in Table 1 . The "Number" in the table indicates the specimen within each tube set, for example, we have tube1-1 to present the first tube specimen in the tube set 1 . After the tube fabrication, we placed all the tubes in the water tank to let the hydrogel beads expand. A minimum of 24 hours is required to have fully expanded hydrogel beads [14]. The fully morphed tubular UISs are shown in Figure 3.

The UISs are usually designed to be submerged or semi-submerged for different applications, which means the structure works underwater or in a humid environment. According to the ASTM D5035[15], the fabric underwater needs to undergo the fabric property test within 15 minutes after being taken out of the water. However, the wet tubular structure may contaminate the bending test equipment. Therefore, we designed a testing platform attached to the water tank to conduct the 3-point bending test. The test platform contains an adjustable frame, tube holders, the force meter, a pulley, and a camera. Figure 4 illustrates the bending test setup; we utilize the 80/20 frames as the main frame which is adjustable to fit the three sets of beams. The test specimen is held by the tube holders at both ends. The holder geometry is designed to cover a semicircle to be capable of holding the tubes in position. Figure 5 demonstrates the tube holder models and corresponding 3D printed assemblies; all the holders are held by shafts connected to the 
brackets, and the brackets are installed on the adjustable frame end. This design allows for the freedom of rotation of supported ends during the bending process.

Table1 Number of hydrogel beads within the tube specimen

\begin{tabular}{llllll}
\hline & Number 1 & Number 2 & Number 3 & Number 4 & Number 5 \\
\hline Tube Set 1 & 140 & 210 & 280 & 350 & 420 \\
Tube Set 2 & 754 & 1130 & 1508 & 1885 & 2262 \\
Tube Set 3 & 1532 & 2298 & 3064 & 3830 & 4596 \\
\hline
\end{tabular}

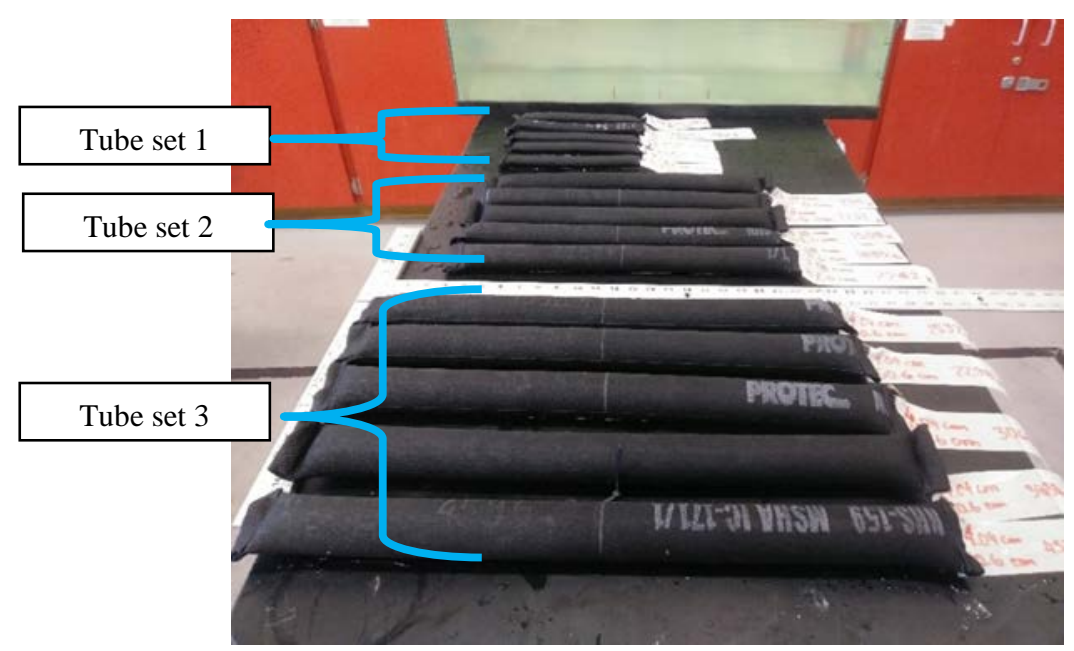

Figure 3. Fully morphed tubes ready for bending test

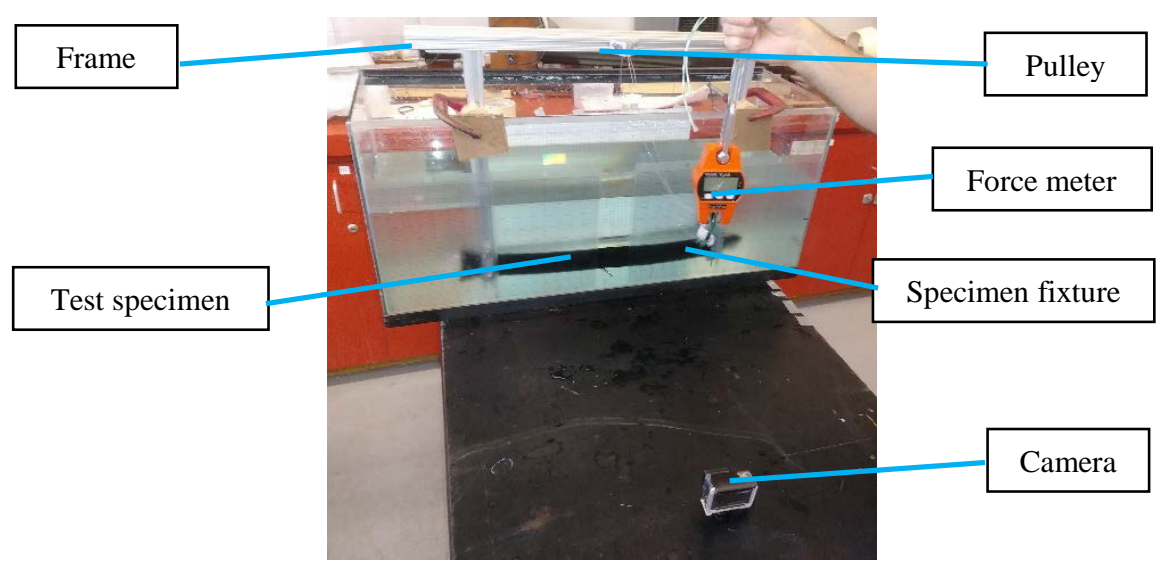

Figure 4. Beam bending test setup

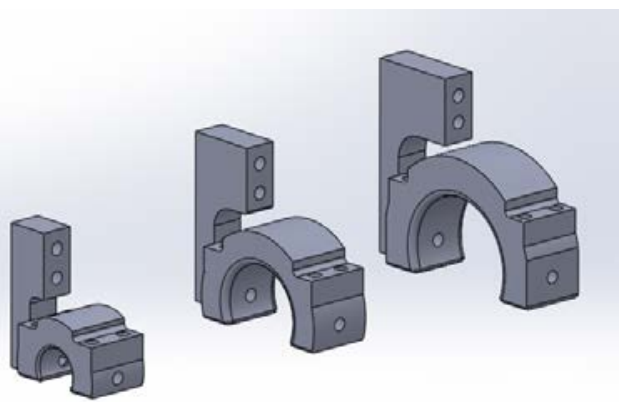

(a) Beam holder 3D models

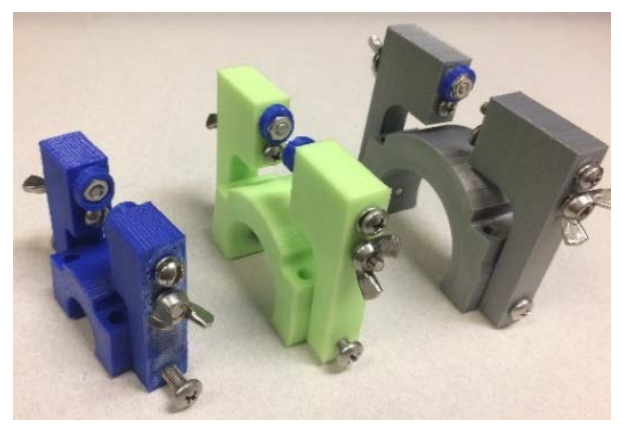

(b) 3D printed beam holder assemblies

Figure 5. Beam holder models and 3D printed assemblies

It is worth mentioning that the diameter of the morphed tube become bigger than its initial size; the morphed tubes dimension details are shown in Table 2. Based on Table 2 data, the fabric of morphed tubes was stretched by the swollen hydrogel beads. It is obvious that more beads within the tube will drive the tube to have a larger diameter. 
During the bending test, the beam specimen was held and one plate was placed at the middle span location to provide the concentrated force. The force is measured by the force meter, and the deflection is recorded by a GoPro camera. The force meter applied in this experiment is the OCSL mini type crane scale with $1 \mathrm{~N}$ accuracy, and the recorded video is $1080 \mathrm{P}$ with 24fps. "Tracker" [16] software was employed for deflection acquisition.

Table 2 Diameters of morphed tubes (units in $\mathrm{mm}$ )

\begin{tabular}{lllllll}
\hline & Initial & Number 1 & Number 2 & Number 3 & Number 4 & Number 5 \\
\hline Tube Set 1 & 18.00 & 21.17 & 22.17 & 22.40 & 22.57 & 22.60 \\
Tube Set 2 & 31.80 & 36.30 & 36.87 & 36.90 & 37.23 & 37.60 \\
Tube Set 3 & 40.40 & 48.00 & 48.57 & 49.1 & 49.97 & 50.57 \\
\hline
\end{tabular}

\section{Results}

We employ Equations (3) and (4) to proceed with the experimental data to obtain the beam flexural stress and flexural strain respectively. Figure 6 presents the three tube sets of flexural stress versus flexural strain curves. We observe that the beams demonstrated different elastic modulus with different levels of beads density. The beams with level 1,2 and 3 beads density exhibited elastic behavior, the beams with level 4 and 5 presented nonlinear elastic modulus. The reason caused this phenomenon is that the bead density effects the hydrogel beads layouts, the beads within high-density level can be squeezed to fracture to turn into another form. The beams with higher level bead density have better flexural properties than the lower one. But there is an optimal bead density for the beams to demonstrate good flexural property, the level 4 beads density is the optimal density the after synthesizing each tube set.

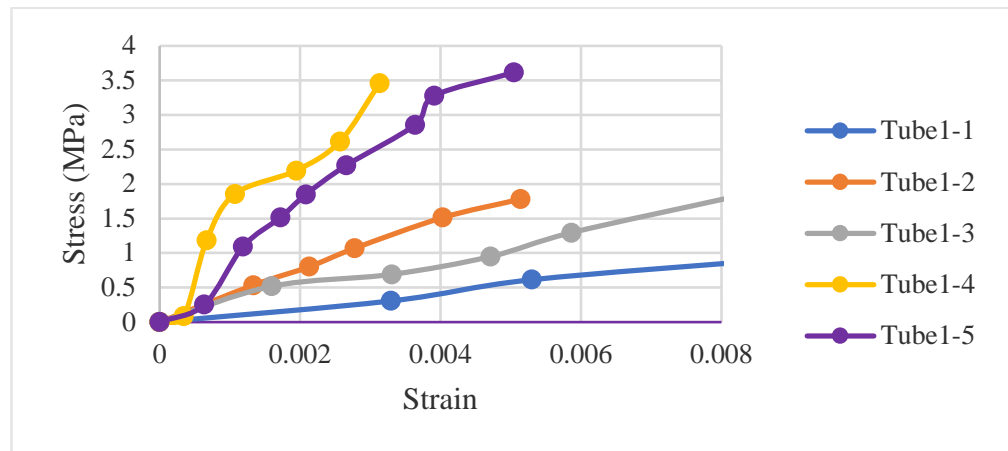

(a) Tube 1 set flexural stress versus flexural strain curve

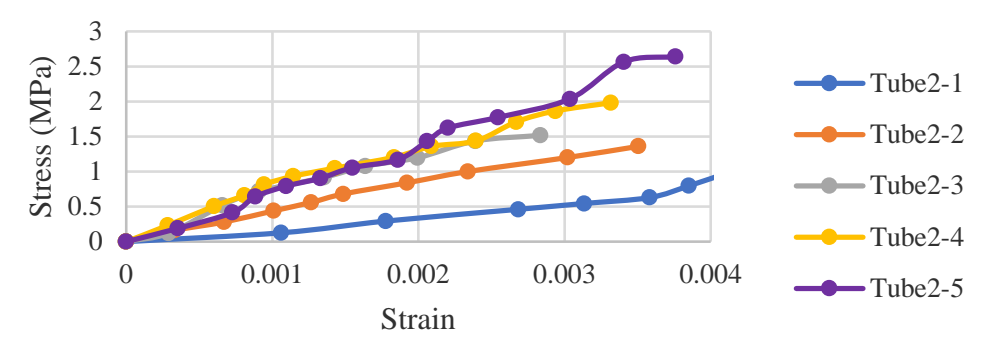

(b) Tube 2 set flexural stress versus flexural strain curve

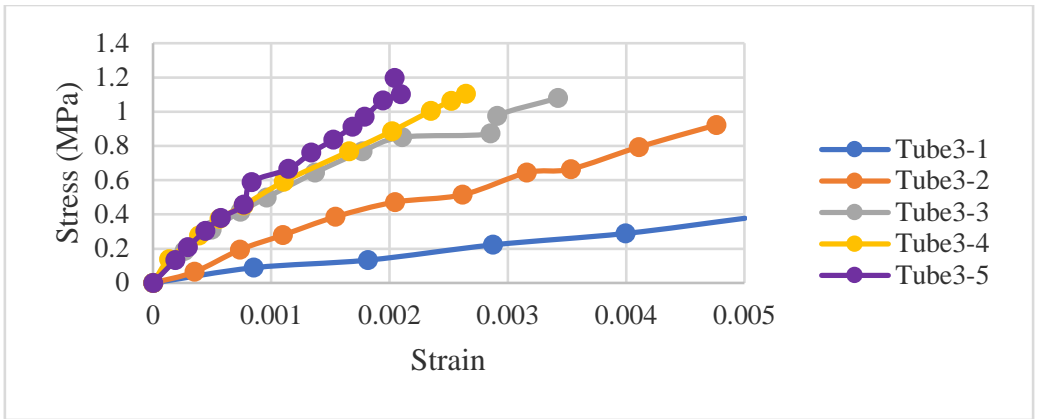

(c) Tube 3 set flexural stress versus flexural strain curve

Figure 6. Tube sets flexural stress versus flexural strain curves 
The elastic modulus of the nylon sleeve is around $17.5 \mathrm{MPa}$ according to the information from the manufacturer. We also compared the value to the online database [17], which is 33.5MPa. Considering the fabric is not a solid material, the nylon sleeve modulus (17.5MPa) is reasonable. Then we calculated the filled material's elastic modulus based on Equation (6) at varying bead densities. The results of the hydrogel beads material modulus within the tubes are shown in Figure 7.

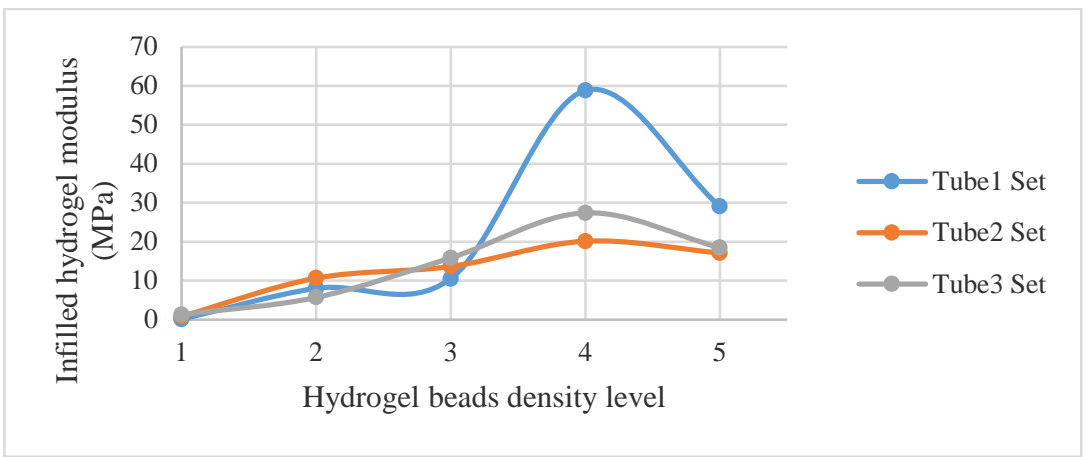

Figure 7. Infilled hydrogel material elastic modulus of different tubes

\section{Discussions and conclusions}

During this study, we calculated the flexural stress and strain for beams with different configurations and the infilled hydrogel modulus values for different beads densities. The results reveal there is an optimal bead density for this type morphed structure to yield the best physical properties. In this research, the optimal bead density is 5 beads $/ \mathrm{cm}^{3}$, which is shown as level 4 in Figure 7. The flexural properties indicate the morphed tubular UIS stiffness, the tube with 5 beads/ $\mathrm{cm}^{3}$ exhibited better flexural property than the others with same length and diameter. It is obvious that the lack of beads leaves spaces within the morphed structure, whereas too many beads can generate higher inner pressure which could smash the beads. The smashed particles do not have the same mechanical properties as the swollen spheres. This is the reason that beams with 6 beads $/ \mathrm{cm}^{3}$ are weaker than the ones with 5 beads/ $\mathrm{cm}^{3}$ density. There is another inference that the beads with highest beads density may not fully grow to the maximum possible sizes. However, the inference is not applicable to our research since the tubes are submerged for weeks before the test, all the beads should have enough time to get in contact with water to swell.

We did not consider the error during the experiment and data processing. The errors include the deflection error and loading error. The deflection measurements were calibrated using a grid which was placed at the front surface of the laboratory water tank with a distance to the tube. This introduced a parallax-type error into the measurement of vertical displacement at the center of the tube. This resulted in an unnecessary error in the measurement of the vertical displacement. The load value is from the force meter with $1 \mathrm{~N}$ accuracy, so during the loading process, we need to apply the tension load by pulling the head of the force meter. This method introduced the error of off-center loads and unstable loads. We will introduce a stepper motor and high accuracy force gauge to apply the loads and acquire the magnitude of the force. This will eliminate the error due to parallax effects and human error during the data acquisition process and the loading process.

In this study, we fabricated three sets of hydrogel bead filled beams with different bead densities and conducted a flexural property experiment. The results verified the hypothesis that there is an optimal bead density for beams with different dimensions. This discovery is critical for this type of composite beams made of hydrogel materials, which establishes a basis for the prediction of tubular UIS properties and also contributes to the UIS designs for future applications.

\section{Acknowledgment}

This work was partially supported by the Harbor Branch Oceanographic Institute (HBOI) foundation Research Excellent fund.

\section{References}

[1] Li Y, Su TC, Ouyang B. Design and deployment analysis of subsea deployable structures. In: The 27th International Ocean and Polar Engineering Conference. International Society of Offshore and Polar Engineers; 2017. 
[2] Ahmed EM. Hydrogel: Preparation, characterization, and applications: A review. Journal of advanced research. 2015;6(2):105-121.

[3] Saxena AK. Synthetic biodegradable hydrogel (PleuraSeal) sealant for sealing of lung tissue after thoracoscopic resection. The Journal of thoracic and cardiovascular surgery. 2010;139(2):496-497.

[4] Singh A, Sharma PK, Garg VK, Garg G. Hydrogels: A review. International Journal of Pharmaceutical Sciences Review and Research. 2010;4(2):97-105.

[5] Chen X, Martin BD, Neubauer TK, Linhardt RJ, Dordick JS, Rethwisch DG. Enzymatic and chemoenzymatic approaches to synthesis of sugar-based polymer and hydrogels. Carbohydrate Polymers. 1995;28(1):15-21.

[6] Slaughter BV, Khurshid SS, Fisher OZ, Khademhosseini A, Peppas NA. Hydrogels in regenerative medicine. Advanced materials. 2009;21(32 - 33):3307-3329.

[7] Zhang L, Li K, Xiao W, Zheng L, Xiao Y, Fan H, Zhang X. Preparation of collagen-chondroitin sulfatehyaluronic acid hybrid hydrogel scaffolds and cell compatibility in vitro. Carbohydrate polymers. 2011;84(1):118-125.

[8] Kashyap NK, Kumar N, Kumar MR. Hydrogels for pharmaceutical and biomedical applications. Critical Reviews $^{\mathrm{TM}}$ in Therapeutic Drug Carrier Systems. 2005;22(2).

[9] L YJ, Xie LQ, Ouyang B, SU TC. Study on material strength of underwater deployable structures. DEStech Transactions on Materials Science and Engineering;2017(ICMSEA_MCE).

[10] https://www.tompkinsind.com/. (Accessed Aug. 20, 2018).

[11] Knaebel A, Rebre SR, Lequeux F. Determination of the elastic modulus of superabsorbent gel beads. Polymer gels and Networks. 1997;5(2):107-121.

[12] Bucciarelli LL. Engineering mechanics for structures. Courier Dover Publications; 2009.

[13] ASTM D790-03, Standard test methods for flexural properties of unreinforced and reinforced plastics and electrical insulating materials, ASTM International, West Conshohocken, PA, 2003, www.astm.org.

[14] Li Y, Ouyang B, Bavar S, Thomas J, Su T, Dalgleish F, Dalgleish A, Zhou T, Ahmad F. Mechanical design consideration of an underwater inflatable co-prime sonar array (UICSA). In: OCEANS2018.

[15] ASTM D5035-11(2015), Standard test method for breaking force and elongation of textile fabrics (strip method), ASTM International, West Conshohocken, PA, 2015, www.astm.org

[16] https://physlets.org/tracker/. (Accessed Aug. 15, 2018).

[17] http://www.matweb.com/search/datasheettext.aspx?matguid=a2e79a3451984d58a8a442c37a226107.(Accessed Sep. 1, 2018).

(C) 2019 by the author(s). This work is licensed under a Creative Commons Attribution 4.0 International License (http://creativecommons.org/licenses/by/4.0/). Authors retain copyright of their work, with first publication rights granted to Tech Reviews Ltd. 